\title{
Placebo-Controlled, Double-Blind Study of Empagliflozin (EMPA) and Implantable Cardioverter-Defibrillator (EMPA- ICD) in Patients with Type 2 Diabetes (T2DM): Rationale and Design
}

Shinya Fujiki $\cdot$ Kenichi Iijima $\cdot$ Masaaki Okabe $\cdot$ Shinichi Niwano $\cdot$ Kenichi Tsujita $\cdot$ Shigeto Naito $\cdot$ Kenji Ando $\cdot$ Kengo Kusano · Ritsushi Kato · Junichi Nitta • Tetsuji Miura • Takeshi Mitsuhashi · Kazuomi Kario •

Yusuke Kondo $\cdot$ Masaki Ieda · Nobuhisa Hagiwara · Toyoaki Murohara $\cdot$ Kazuyoshi Takahashi $\cdot$ Hirofumi Tomita Yasuchika Takeishi · Toshihisa Anzai · Wataru Shimizu • Masafumi Watanabe $\cdot$ Yoshihiro Morino · Takeshi Kato • Hiroshi Tada - Yoshihisa Nakagawa - Masafumi Yano - Koji Maemura - Takeshi Kimura · Hisako Yoshida • Keiko Ota - Takahiro Tanaka • Nobutaka Kitamura • Koichi Node • Yoshifusa Aizawa • Ippei Shimizu • Daisuke Izumi · Kazuyuki Ozaki · Tohru Minamino (D) on behalf of the EMPA-ICD investigators

Received: April 9, 2020 / Accepted: September 3, 2020 / Published online: September 23, 2020 (C) The Author(s) 2020

\section{ABSTRACT}

Introduction: Type 2 diabetes (T2DM) is associated with cardiovascular death, including sudden cardiac death due to arrhythmias. Patients with an implantable cardioverter-

Digital Features This article is published with digital features to facilitate understanding of the article. To view digital features for this article go to https://doi.org/ 10.6084/m9.figshare.12827783.

Ihe EMPA-ICD investigators are listed in acknowledgements.

Electronic Supplementary Material The online version of this article (https://doi.org/10.1007/s13300020-00924-9) contains supplementary material, which is available to authorized users.

S. Fujiki · K. Iijima - I. Shimizu - D. Izumi · K. Ozaki · T. Minamino ( $\square)$

Department of Cardiovascular Biology and

Medicine, Niigata University Graduate School of

Medical and Dental Sciences, Niigata, Japan

e-mail: t.minamino@juntendo.ac.jp

M. Okabe · Y. Aizawa

Department of Cardiology, Tachikawa General

Hospital, Nagaoka, Niigata, Japan defibrillator (ICD) are also at high risk of developing a clinically significant ventricular arrhythmia. It has been reported that sodium-glucose cotransporter 2 (SGLT2) inhibitors can reduce cardiovascular deaths; however, the physiological mechanisms of this remain unclear. It is, however, well known that SGLT2 inhibitors increase blood ketone bodies, which have been suggested to have sympathosuppressive effects. Empagliflozin (EMPA) is an SGLT2 inhibitor. The current clinical trial titled "Placebo-controlled, double-blind study of empagliflozin (EMPA) and implantable cardioverter-defibrillator (EMPA-ICD) in patients with type 2 diabetes (T2DM)" was designed to investigate the antiarrhythmic effects of EMPA.

\footnotetext{
S. Niwano

Cardiovascular Medicine, Kitasato University,

Sagamihara, Kanagawa, Japan

K. Tsujita

Department of Cardiovascular Medicine, Graduate School of Medical Sciences, Kumamoto University, Kumamoto, Japan
} 
Methods: The EMPA-ICD study is a prospective, multicenter, placebo-controlled, double-blind, randomized, investigator-initiated clinical trial currently in progress. A total of 210 patients with T2DM (hemoglobin A1c 6.5-10.0\%) will be randomized $(1: 1)$ to receive once-daily placebo or EMPA, $10 \mathrm{mg}$, for 24 weeks. The primary endpoint is the number of clinically significant ventricular arrhythmias for 24 weeks before and 24 weeks after study drug administration, as documented by the ICD. The secondary endpoints of the study are the change from baseline concentrations in blood ketone and catecholamine 24 weeks after drug treatment.

Conclusion: The EMPA-ICD study is the first clinical trial to assess the effect of an SGLT2 inhibitor on clinically significant ventricular arrhythmias in patients with T2DM and an ICD. Trial registration: Unique trial number, jRCTs031180120 (https://jrct.niph.go.jp/latestdetail/jRCTs031180120).

Keywords: Arrhythmia; Empagliflozin (EMPA); Implantable cardioverter-defibrillator (ICD); Ketone bodies; Sympathetic nerve activity; Type 2 diabetes (T2DM)

S. Naito

Division of Cardiology, Gunma Prefectural

Cardiovascular Center, Maebashi, Gunma, Japan

K. Ando

Department of Cardiology, Kokura Memorial

Hospital, Kitakyushu, Fukuoka, Japan

K. Kusano

Department of Cardiovascular Medicine, National Cerebral and Cardiovascular Center, Suita, Osaka, Japan

R. Kato

Department of Cardiology, Saitama Medical

University International Medical Center, Hidaka,

Saitama, Japan

R. Kato

Department of Arrhythmia, Saitama Medical

University International Medical Center, Hidaka,

Saitama, Japan

\section{Key Summary Points}

Type 2 diabetes mellitus is associated with a high risk of atherosclerosis and subsequent cardiovascular events, and an increase in life-threatening arrhythmia events, but little data is currently available regarding therapeutic strategies to improve prognosis in these patients.

The recent EMPA-REG OUTCOME study showed that administration of the sodium-glucose transporter 2 (SGLT2) inhibitor empagliflozin (EMPA) significantly suppressed cardiovascular death including sudden cardiac death, but the mechanisms underlying the beneficial effects of EMPA remain unknown.

It is well known that SGLT2 inhibitors increase blood ketone bodies, which have been suggested to have sympathosuppressive effects. We therefore designed the current clinical trial titled "Placebocontrolled, double-blind study of empagliflozin and implantable cardioverter-defibrillator (EMPA-ICD) in patients with type 2 diabetes" to investigate the antiarrhythmic effects of EMPA.

J. Nitta

Department of Cardiology, Sakakibara Heart Institute, Fuchu, Tokyo, Japan

T. Miura

Department of Cardiovascular, Renal and Metabolic Medicine, Sapporo Medical University School of Medicine, Sapporo, Hokkaido, Japan

T. Mitsuhashi

Department of Cardiology, Jichi Medical University Saitama Medical Center, Saitama, Japan

K. Kario

Division of Cardiovascular Medicine, Department of Medicine, Jichi Medical University School of Medicine, Shimotsuke, Tochigi, Japan

Y. Kondo

Department of Cardiovascular Medicine, Chiba University Graduate School of Medicine, Chiba, Japan 


\section{INTRODUCTION}

An implantable cardioverter-defibrillator (ICD) is one of the most effective and established treatments in the improvement of the prognosis of life-threatening arrhythmias and the prevention of sudden cardiac death due to lifethreatening arrhythmias [1-11]. Several therapeutic guidelines for cardiac arrhythmias recommend ICD implantation for patients with life-threatening arrhythmias irrespective of the type of heart disease $[12,13]$. Patients with an ICD also receive general medical treatment such as beta-blockers for arrhythmias or heart failure, to reduce life-threatening arrhythmic events. However, an ICD shock is also strongly associated with a poor prognosis [14, 15], worsened physical functioning, and mental well-being [16].

Type 2 diabetes mellitus (T2DM) is associated with a high risk of atherosclerosis and subsequent cardiovascular (CV) events [17, 18], and an increase in life-threatening arrhythmia events [19]. Therefore, T2DM is an important

\footnotetext{
M. Ieda

Department of Cardiology, Faculty of Medicine, University of Tsukuba, Tsukuba, Ibaraki, Japan

N. Hagiwara

Department of Cardiology, Tokyo Women's Medical University, Tokyo, Japan

T. Murohara

Department of Cardiology, Nagoya University Graduate School of Medicine, Nagoya, Aichi, Japan

K. Takahashi

Department of Cardiology, Niigata City General

Hospital, Niigata, Japan

H. Tomita

Department of Cardiology and Nephrology, Hirosaki University Graduate School of Medicine, Hirosaki, Aomori, Japan

Y. Takeishi

Department of Cardiovascular Medicine, Fukushima Medical University, Fukushima, Japan

T. Anzai

Department of Cardiovascular Medicine, Hokkaido University Graduate School of Medicine, Sapporo, Hokkaido, Japan
}

therapeutic target in patients with an ICD. Currently, little data is available regarding therapeutic strategies to improve prognosis in these patients. The recent EMPA-REG OUTCOME study showed that administration of the sodium-glucose transporter 2 (SGLT2) inhibitor empagliflozin (EMPA) significantly suppressed cardiovascular death, including sudden cardiac death [20]. This study reported pleiotropic effects, including hypotension and body weight reduction, as a result of EMPA administration. The physiological mechanisms involved in EMPA administration and cardiac function, however, remain unknown.

Following on from findings of previous studies [21] the current study will focus on increases in blood concentrations of ketone bodies after administration of EMPA. Ketone bodies are produced primarily in the liver and have been reported to alleviate oxidative stress and/or suppress sympathetic nerve activity in tissues $[22,23]$. It has therefore previously been hypothesized that ketone bodies may suppress clinically significant ventricular arrhythmic
W. Shimizu

Department of Cardiovascular Medicine, Nippon Medical School, Tokyo, Japan

M. Watanabe

Department of Cardiology, Pulmonology, and Nephrology, Yamagata University Faculty of Medicine, Yamagata, Japan

\section{Y. Morino}

Division of Cardiology, Department of Internal Medicine, Iwate Medical University, Morioka, Iwate, Japan

\section{T. Kato}

Department of Cardiology, Graduate School of Medical Science, Kanazawa University, Kanazawa, Ishikawa, Japan

H. Tada

Department of Cardiovascular Medicine, Faculty of Medical Sciences, University of Fukui, Fukui, Japan

Y. Nakagawa

Department of Cardiovascular Medicine, Shiga University of Medical Science, Otsu, Shiga, Japan 
events such as non-sustained ventricular tachycardia (NSVT), ventricular tachycardia (VT), or ventricular fibrillation (VF). On the basis of this rationale, the primary objective of the present study is to evaluate the rate of arrhythmic events in patients with T2DM with an ICD before and after treatment with EMPA. In addition, the levels of ketone bodies and catecholamines will be assessed to further investigate their relationship with arrhythmic events in T2DM.

It is anticipated that the present study will contribute to not only understanding the physiological effects of EMPA but also establish improved therapy for patients with cardiac disorders complicated by T2DM.

\section{METHODS}

\section{Study Overview and Design}

The EMPA-ICD study is a prospective, multicenter, placebo-controlled, double-blind randomized, investigator-initiated clinical trial. A total of 210 patients with T2DM (hemoglobin

\footnotetext{
M. Yano

Division of Cardiology, Department of Medicine and Clinical Science, Yamaguchi University

Graduate School of Medicine, Yamaguchi, Japan

K. Maemura

Department of Cardiovascular Medicine, Nagasaki

University Graduate School of Biomedical Sciences,

Nagasaki, Japan

T. Kimura

Department of Cardiovascular Medicine, Kyoto

University Graduate School of Medicine, Kyoto, Japan

H. Yoshida

Department of Medial Statistics, Osaka City

University, Graduate School of Medicine, Osaka, Japan

K. Ota

Data Management Group, Department of Clinical Research Support, Center for Clinical Research and Innovation, Osaka City University Hospital, Osaka, Japan
}

A1c [HbA1c] 6.5-10.0\%) and an ICD will be allocated to (1:1) EMPA (10 mg once daily) (Boehringer Ingelheim, Ingelheim am Rhein, Germany) or placebo, for 24 weeks under double-blinded conditions. Patients will undergo evaluation of the ICD during the 24 weeks before treatment and the 24 weeks after treatment to determine if EMPA can improve the number of clinically significant ventricular arrhythmia events. The study protocol was reviewed and approved by the Certified Review Board of the Niigata University Graduate School of Medicine (certification no. CRB3180025) and registered by the jRCT in February 2019 (ID jRCTs031180120). This study is being conducted in accordance with the guidelines of the Declaration of Helsinki and in compliance with the Clinical Trial Act, a Japanese law designed to ensure that researchers monitor, adhere to the study criteria, and assess and manage any conflicts of interest. After the initial screening, each patient was given a thorough explanation of the trial plan, and before inclusion in the study, each patient provided signed informed consent forms.

\section{T. Tanaka $\cdot$ N. Kitamura}

Clinical and Translational Research Center, Niigata University Medical and Dental Hospital, Niigata, Japan

\section{K. Node}

Department of Cardiovascular Medicine, Saga

University, Saga, Japan

I. Shimizu

Divisiont of Molecular Aging and Cell Biology,

Niigata University Graduate School of Medical and Dental Sciences, Niigata 951-8510, Japan

T. Minamino

Department of Cardiovascular Biology and

Medicine, Juntendo University Graduate School of

Medicine, Tokyo 113-8421, Japan

T. Minamino

Japan Agency for Medical Research and

Development-Core Research for Evolutionary

Medical Science and Technology (AMED-CREST),

Japan Agency for Medical Research and

Development, Tokyo, Japan 
Table 1 Inclusion and exclusion criteria

Inclusion
1. $\geq 20$ years of age
2. ICD or CRT-D implantation
surgery $>24$ weeks before eligibility test
3. T2DM with HbAlc $\geq 6.5$ to $\leq 10 \%$
4. Signed, written informed consent to
participate in the study

\section{Exclusion}

1. Patients using an SGLT2 inhibitor at the time of the eligibility test

2. Patients who received an SGLT2 inhibitor within 24 weeks before the eligibility test

3. Patients with a history of hypersensitivity to EMPA

4. Patients with a history of diabetic ketoacidosis, diabetic coma, or hypoglycemic episodes

5. Patients with severe infections, upcoming surgery, or seriously injured at the time of eligibility tests

6. Type 1 diabetes

7. New York Heart Association Class IV heart failure

8. Severe renal dysfunction (eGFR $<30 \mathrm{~mL} / \mathrm{min} / 1.73 \mathrm{~m}^{2}$ or dialysis)

9. Serious hepatic function disorder (aspartate transaminase or alanine transaminase $>3$ times higher than the institutional standards)

10. Pituitary or adrenal insufficiency

11. Malnutrition or fasting condition

12. History of excessive alcohol intake

13. Gastrointestinal disorder such as diarrhea or vomiting

14. Urinary tract or genital infection

15. Pregnant or suspected pregnancy

16. Body mass index $\leq 18.5 \mathrm{~kg} / \mathrm{m}^{2}$

17. Patients with the following events within 24 weeks before the eligibility test: alteration of antiarrhythmic drug; catheter ablation for ventricular arrhythmia; coronary revascularization; open-heart surgery; development of coronary artery disease, stroke or transient ischemic stroke; seizure; infection requiring hospitalization, or cardiac failure requiring hospitalization

18. ICD unable to record NSVT

19. Non-remitted malignant tumor

\section{Trial Population and Recruitment}

A total of 210 patients will be recruited across 28 institutions in Japan from April 2019 to April 2021. The inclusion and exclusion criteria for enrollment are shown in Table 1. Briefly, eligible patients include those with T2DM (HbA1c 6.5-10.0\%), and with an ICD for more than 24 weeks before commencement of the study. Patients in whom the following events were considered to be likely to affect the onset of clinically significant ventricular arrhythmia 


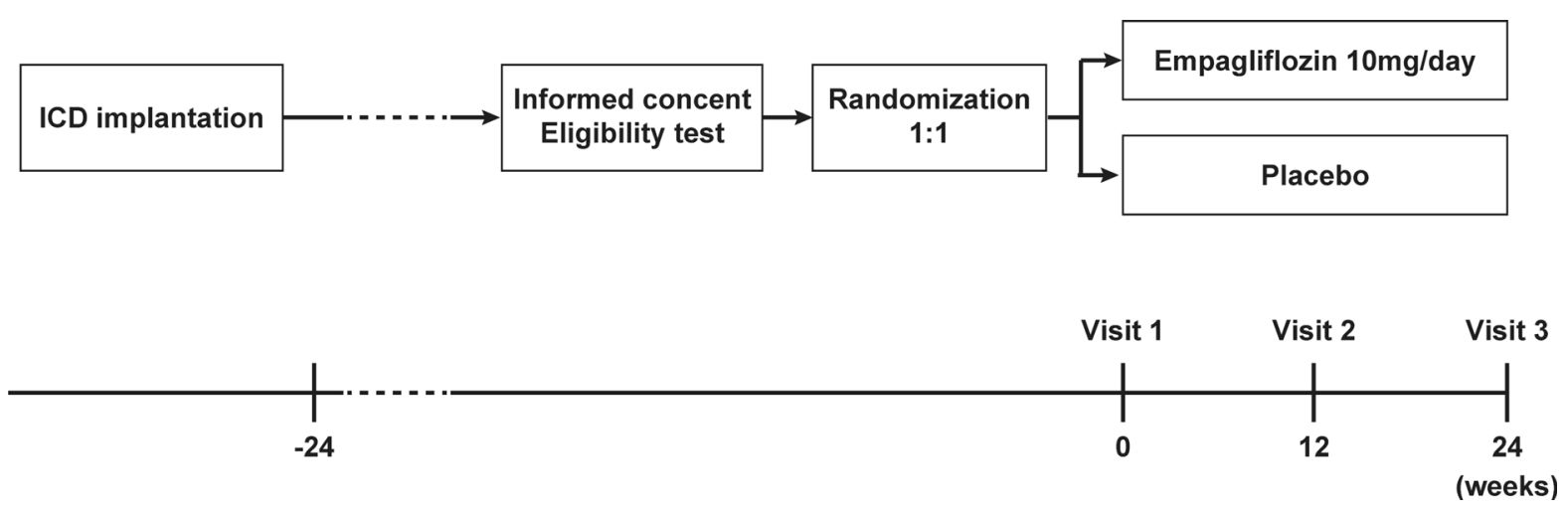

Fig. 1 Study outline. Implantable cardioverter-defibrillator (ICD)

within 24 weeks before the eligibility tests will be excluded: alteration of antiarrhythmic drug; catheter ablation for ventricular arrhythmia; coronary revascularization; open-heart surgery; development of coronary artery disease, stroke or transient ischemic stroke; seizure; infection requiring hospitalization, and cardiac failure requiring hospitalization. After initial screening, each patient will receive an explanation of the trial plan and will be asked to complete a written informed consent form.

\section{Trial Design and Follow-up}

Patients will be randomized to receive either EMPA or placebo (10 mg once daily) with follow-up visits scheduled at 12 and 24 weeks after treatment (Fig. 1). Each patient will receive standard treatment for their background disease in addition to administration of the study drug.

\section{Randomization, Data Collection, and Blinding}

After the patient's eligibility is confirmed, randomization (EMPA or placebo, $10 \mathrm{mg}$ once daily) will be performed by the stratified allocation method using the following factors: left ventricular ejection fraction (LVEF) $(<40 \%$ or $\geq 40 \%$ ), age ( $<65$ or $\geq 65$ years), and gender. The study will be performed in a double-blind manner until completion of data fixation. Randomization and data collection will be performed using the Research Electronic Data
Capture (REDCap) [24, 25], EDC method which is a web-based software platform designed to support data capture for research studies. Briefly, patients are registered and designated with an identification number and study drug identification number, thereby ensuring personal information protection and double-blind conditions. Data will be managed by the independent organization of Osaka City University until the end of the study.

To maintain the double-blinding of the study, laboratory tests (e.g., urine glucose tests and urinary ketone body tests) that may distinguish whether the study drug or placebo has been assigned, and questions about changes in urine volume, will be avoided as much as possible. The blinding codes will be opened only under emergency situations where appropriate medical treatment is necessary for serious adverse events (AEs) or the patient's safety. When the blinding code is opened, it will be immediately reported to the Study-Representing Physician, and the patient's participation in the study will be discontinued.

\section{Treatment}

All patients will receive EMPA or placebo (10 mg once daily) and be followed for 24 weeks. If investigators decide that the blood glucose level of a patient is not properly controlled, they will be allowed to add and discontinue diabetes drugs and increase or decrease the dose of such drugs, including insulin; no changes to EMPA 
will be permitted, and the daily dose of EMPA should remain fixed at $10 \mathrm{mg} /$ day. Adverse reactions such as hypoglycemia or dehydration will be monitored. The therapeutic treatment guidelines for T2DM are based on the Treatment Guide for Diabetes in Japan [26]. The initiation, choice, and dosage of these drugs are at the investigator's discretion. Patients can also receive treatment for arrhythmias or underlying heart disease. Changes in antiarrhythmic drugs such as beta-blockers and ion channel blockers will be avoided if possible during the study. If changes in antiarrhythmic drugs do occur after enrollment, the patient's participation in the study will be discontinued as these drugs may affect the frequency of clinically significant ventricular arrhythmic events. After completion of the treatment period, treatment will be continued with optional diabetes drugs.

\section{Measurements}

Baseline characteristics including height; gender; age; smoking history, alcohol drinking history; clinically significant ventricular arrhythmias that caused the original implantation of the ICD and other underlying diseases; manufacturer and type of ICD; current and past comorbidities; and history of drug and nondrug treatment will be noted when subject eligibility is determined. Background treatments and physical examination will be performed at each visit. Investigators will comply with the study protocol, report AEs after informed consent is provided, and any study drug non-compliance by patients after drug administration begins. Body weight and body temperature will be measured when subject eligibility is determined and at 0 and 24 weeks. The following data will be investigated at 0 and 24 weeks: blood pressure; pulse rate; blood tests (e.g., blood count, biochemical markers); 12-lead electrocardiogram (ECG); Holter monitoring; and echocardiography (Table 2). These tests will be performed at local institutes. Specific blood tests will include acetoacetic acid; 3-hydroxybutyric acid; total ketone bodies; adrenaline; noradrenaline; dopamine; erythropoietin; and reticulocytes and will be conducted at 0 and
24 weeks. These specific blood tests will be measured at central laboratories. All blood tests at 0 and 24 weeks will be conducted under fasting conditions with subjects resting in a supine position on a bed for no less than 30 min. Patients will have the option of undergoing ${ }^{123}$ I-metaiodobenzylguanidine (MIBG) scintigraphy at 0 and 24 weeks. The ICD will be investigated at 0 and 24 weeks to evaluate clinically significant ventricular arrhythmic events.

\section{Measurement of Clinically Significant Ventricular Arrhythmic Events}

Clinically significant ventricular arrhythmic events recorded in the ICD will be evaluated at 0 and 24 weeks. At 0 weeks, arrhythmic events that occurred from 24 weeks before treatment to 0 weeks will be recorded. At 24 weeks after commencement of the study, arrhythmic events that occurred from 0 to 24 weeks will be recorded. In this study, clinically significant ventricular arrhythmic events will be categorized as NSVT, VT, or VF depending on the judgment noted by the defibrillators and verification by members of the Event Assessment Committee. Device settings for detection and treatment of arrhythmias are at the discretion of each physician. Device reports including the list of arrhythmic events and intracardiac ECGs will be obtained by individual interviews at outpatient clinics or through a remote monitoring system. During the study, changes in device settings will only be permitted if the physicians judge it necessary to avoid malfunction of the ICD.

\section{Trial Endpoints}

The primary endpoint of this study is the change in the frequency of clinically significant ventricular arrhythmic events from 0 to 24 weeks. Secondary endpoints are changes in the following parameters from 0 to 24 weeks: number of clinically significant ventricular arrhythmic events and appropriate device operation; change in parameters obtained via Holter monitoring; changes in blood ketone 
Table 2 Follow-up schedule

\begin{tabular}{|c|c|c|c|c|}
\hline & Eligibility test & 0 week & 12 weeks & 24 weeks \\
\hline Patient information/obtaining consent & a & & & \\
\hline Patient background & a & & & \\
\hline Interview/physical examination & a & a & a & a \\
\hline Confirmation of drug compliance & & & a & a \\
\hline Confirmation of study procedure compliance & & a & a & a \\
\hline Confirmation of concomitant drug use & a & a & a & a \\
\hline Height & $\mathrm{a}$ & & & \\
\hline Body weight/body temperature & a & a & $\mathrm{b}$ & a \\
\hline Blood pressure/pulse rate & & a & $\mathrm{b}$ & a \\
\hline Blood tests & a & a & $\mathrm{b}$ & a \\
\hline Blood tests (specific) & & a & $\mathrm{b}$ & a \\
\hline 12-lead ECG & & a & & a \\
\hline Holter monitoring & & a & & a \\
\hline Echocardiography & a & a & & a \\
\hline${ }^{123}$ I-MIBG myocardial scintigraphy & & $\mathrm{b}$ & & $\mathrm{b}$ \\
\hline Safety assessment & a & a & a & a \\
\hline Evaluation of ICD & & a & & a \\
\hline
\end{tabular}

${ }^{a}$ Primary or secondary outcome measure

b Optional

fractions (acetoacetic acid, 3-hydroxybutyric acid, and total ketone bodies), and changes in blood catecholamine fractions (adrenaline, noradrenaline, and dopamine). In addition to these endpoints, exploratory endpoints will also be investigated including change in body weight; body temperature; blood pressure; pulse rate; general blood tests; 12-lead ECG; ECG parameters; specific blood tests (erythropoietin and reticulocytes); heart-to-mediastinum ratio; and washout rate data obtained via ${ }^{123}$ I-MIBG scintigraphy (if obtained).

\section{Safety}

To evaluate the patients' safety, all AEs will be reported during the study. To ensure compliance with Japanese law (the Clinical Trials Act), AEs related to the trial drugs and study protocol will be reported. Moreover, adverse events of special interest such as hepatic injury, decreased renal function, metabolic acidosis, ketoacidosis, diabetic ketoacidosis, lower limb amputation, and Fournier's gangrene will be clearly defined and reported. When investigators report AEs, the onset date, severity, treatment, outcome, and causal relationship to the study drug and protocol will be reported. This information will be shared immediately with the secretariat or principal investigator by REDCap and will be reported to Nippon Boehringer Ingelheim and the Independent Monitoring Committee (IDMC). The IDMC consists of diabetologists and cardiologists who 
independently judge the safety and the necessity for any revisions and study discontinuation due to AEs.

\section{Sample Size Estimation}

As a result of the pilot character of this study and lack of research studies directly comparable to ours, we prepared the available 200 samples on the basis of effect size for a future primary outcome. We estimated that this sample size for a total of each group would provide the trial with a power of $80 \%$ at the $5 \%$ significance level to detect an effect size of 1.44 as the incidence rate ratio (IRR) of arrhythmias in the placebo group compared with the EMPA group on the basis of a previous study [27]. It is anticipated that any beneficial effects EMPA has on sympathetic nervous activity will provide considerable improvements in patients with heart disease. Clinically, one of the most beneficial outcomes in the reduction of sympathetic nervous activity is a decrease in cardiac arrhythmias. In patients with T2DM, the sympathetic counter-regulatory response to hypoglycemia by systemic adrenaline and noradrenaline release [28] as well as enhanced sympathetic neural activity [29] contributes to the occurrence of arrhythmic events [27]. It is therefore hypothesized that the risk of hypoglycemia to sympathetic nervous activity is equal to the disadvantage observed in SGLT2 inhibitor therapy-naïve subjects with T2DM. The IRR of 1.44 from this previous study was consequently used to justify the above sample number, as no previous studies have examined the frequency of VT, VF, and NSVT in ICD patients receiving an SGLT2 inhibitor.

\section{Statistical Analysis}

The analyses of study endpoints will be performed in the full analysis set. Continuous variables in the text and tables are expressed as means \pm standard deviation, and categorical variables are expressed as numbers (percentages). Patient characteristics will be compared using chi-square tests for categorical variables, $t$ tests will be used for normally distributed continuous variables, and the Wilcoxon rank sum tests will be used for continuous variables with a skewed distribution.

The number of clinically significant ventricular arrhythmic events recorded in the ICD will be evaluated at weeks 0 and 24 (or study discontinuation), and subjected to Poisson regression analysis by the generalized linear model, assigning difference in the number of severe arrhythmic events between the EMPA and placebo groups as an objective variable.

Descriptive summary statistics will be calculated for each endpoint. For continuous variables, differences will be estimated on the basis of the least-squares method. For discrete variables, differences in the ratio will be estimated on the basis of chi-square distribution. Other analyses are shown in the detailed statistical plan provided elsewhere.

All $p$ values will be two-sided, and $p<0.05$ will be considered statistically significant. All statistical analyses will be performed using SAS version 9.4 (SAS Institute, Cary, NC, USA).

\section{Trial Organization and Oversight}

The principal investigator of the EMPA-ICD is Tohru Minamino from the Department of Cardiovascular Biology and Medicine Niigata University Graduate School of Medical and Dental Sciences. The research advisor is Koichi Node from the Department of Cardiovascular Medicine at Saga University. The steering committee will manage the planning, operational, analytical, and presentation aspects of the study. The IDMC will manage the safety section. The Event Evaluation Committee will confirm reported arrhythmias. The trial secretariats are in the Department of Cardiovascular Biology and Medicine Niigata University Graduate School of Medical and Dental Sciences and Micron Inc., Tokyo, Japan. The trial drugs, provided by Boehringer Ingelheim, are to be stored appropriately at the Department of Clinical and Translational Center at Niigata University Hospital, and will be distributed to each institute depending on patient registrations as recorded on clinical report forms on the web site. Data management, monitoring 
activities, statistical analyses, and audits will be performed independently on the basis of an outsourcing agreement.

\section{DISCUSSION}

The prospective, multicenter, placebo-controlled, double-blind, randomized, investigatorinitiated EMPA-ICD clinical trial is in progress to study the effect of EMPA on clinically significant ventricular arrhythmias in patients with T2DM and an ICD. The number of clinically significant ventricular arrhythmias during 24 weeks before and after study drug administration, as documented by the ICD, will be compared between the active-control group and placebo-control groups.

The prevalence of T2DM, a metabolic disease, is approximately $8.5 \%$ of the world's population, and this number is expected to increase in the future [30]. The risk for cardiovascular disease and death increases 2-3 times in patients with T2DM [31, 32]. Among cardiovascular diseases, not only coronary artery diseases [33-37] but also non-coronary diseases such as microangiopathy and autonomic nerve disorders [38, 39] have been suggested to be associated with sudden cardiac death. Ventricular arrhythmias such as VT and VF are presumed to be the major cause of such sudden death. Diabetes and arrhythmias are assumed to be closely related; however, the extent and underlying mechanism of this relationship remain unclear. Therefore, it is important to investigate this relationship to improve the outcome of patients with T2DM.

\section{Importance of Examining Arrhythmias}

Assessment of clinically significant ventricular arrhythmias is important to elucidate the mechanism underlying the potential impact of EMPA in patients with T2DM at risk of cardiovascular disease. The EMPA-REG OUTCOME trial [20], the CANVAS trial [40], and the DECLARE-TIMI 58 trial [41] demonstrated favorable effects of SGLT2 inhibitors in not only mortality but also cardiovascular outcomes. In the supplemental data of the EMPA-REG
OUTCOME study, both "sudden death" (placebo vs. $\mathrm{EMPA}=38$ [1.6\%] vs. 53 [1.1\%]) and "other cardiovascular death" (placebo vs. $\mathrm{EMPA}=55[2.4 \%]$ vs. $74[1.6 \%])$ tended to be less in the EMPA group, although these data were not statistically verified. Thus, this tendency for reduced cardiac events may be partially explained by the reduction of clinically significant ventricular arrhythmias by EMPA.

Moreover, all three cardiovascular outcomes trials consistently indicated the benefit of SGLT2 inhibitors including EMPA in the reduction of hospitalization for heart failure. One hypothesis is that this outcome is due to the diuretic effect of SGLT2 inhibitors in general. It has been shown that SGLT2 inhibitors induce osmotic diuresis and natriuresis by decreasing the reabsorption of glucose and sodium, resulting in less extracellular volume; a possible reduction in vascular wall stress; improved cardiac function; and potentially reduced congestion [42]. One important fact associated with the diuretic effect of SGLT2 inhibitors is the lack of changes in serum potassium levels [43]. It is well known that abnormal serum potassium levels affect the cardiac repolarization and can induce lifethreatening arrhythmias [44]. In comparison with classic diuretics, the lack of impact on serum potassium levels by SGLT2 inhibitors may minimize the incidence of life-threatening arrhythmias. Indeed, in humans, it has been shown that SGLT2 inhibitors do not prolong the QT interval during cardiac repolarization [45]. As these study results do not provide sufficient evidence of an antiarrhythmic quality of EMPA, it therefore remains important to determine if EMPA can regulate body fluid volume without increasing arrhythmic events. Therefore, the primary endpoint of this current study may create a novel insight in regards to the mechanism of action of EMPA.

\section{How to Accurately Assess Arrhythmias}

In the present study, arrhythmias will be assessed by ICD information before and after drug administration. This study is designed to evaluate the effect of EMPA on arrhythmic events 
correctly. First, arrhythmias in same patient will be compared and analyzed to minimize confounding factors, such as differences in ICD models and underlying heart disease. Second, during the 24 weeks after study drug administration, the ICD setting will not be changed from before the study as changes in the ICD setting can significantly impact the detection of arrhythmias. Third, patients with problems that can affect the number of arrhythmias, including alteration of antiarrhythmic drugs; catheter ablation for ventricular arrhythmias; coronary revascularization; open-heart surgery; development of coronary artery disease, stroke, or transient ischemic stroke; seizure; infection requiring hospitalization; or cardiac failure requiring hospitalization will be excluded at the time of study initiation. If these problems occur during the study, patients will be withdrawn from the study. Finally, the Event Evaluation Committee is an external organization and will be asked to evaluate arrhythmias recorded in the ICD and confirm that the primary endpoints are correctly reported.

\section{Validity of the Definition of Clinically Significant Ventricular Arrhythmia}

In the present study, a clinically significant ventricular arrhythmia is defined as VF, VT, and/or NSVT. Generally, in the setting of an ICD, anti-tachycardia therapy includes delivering a shock for VF and VT, whereas only monitoring is performed for NSVT. However, NSVT increases the risk for VT and VF. Several reports have indicated that NSVT is a criterion for ICD implantation. In the MADIT-I study, performed to investigate the effect of ICD on patients with myocardial infarction, NSVT was an inclusion criterion [1]. In patients with NSVT with ischemic heart disease with LVEF $\leq 40 \%$, electrophysiologic examination showed that use of an ICD increased survival rates [46]. Therefore, a primary endpoint in this present study that includes NSVT is deemed to be appropriate for the evaluation of clinically significant ventricular arrhythmias.

\section{Importance of Secondary Evaluation Items}

Blood concentrations of ketone bodies and catecholamines will be used as secondary endpoints in this study. To minimize variables, these markers will be measured with subjects in the fasting state and resting. Recently, ketone bodies have gained attention as one of the possible explanations for the underlying mechanism of the favorable effect of SGLT2 inhibitors on CV outcome [47]. Generally, in the fasting state, production of ketone bodies, called "ketogenesis," occurs in the mitochondria of the liver. Ferrannini et al. reported that the SGLT2-induced increase in lipid mobilization and oxidative use were associated with increased levels of plasma $\beta$-hydroxybutyrate $(\beta \mathrm{OHB})$, which is a type of ketone body [21]. Among the ketone bodies, acetoacetate and $\beta \mathrm{OHB}$ reach the systemic organs and are used as an energy source [22]. In particular, ketone bodies with good metabolic efficiency are used instead of glucose and fatty acids, which may have a positive impact on the failing heart $[48,49]$. In addition, $\beta$ OHB has been found to function as a ligand involved in various signals. Won et al. reported that $\beta \mathrm{OHB}$ plays a potentially important role in regulating the activity of the sympathetic nervous system [23]. It is considered that increased $\beta \mathrm{OHB}$ levels may reduce the occurrence of arrhythmias, as $\beta \mathrm{OHB}$ may act as a sympatho-suppressive agent. By using ketone bodies and catecholamine levels as secondary endpoints for the present study, it is expected that the underlying mechanism of any favorable effect of EMPA will be reinforced.

The current EMPA-ICD is a valuable research study that may provide a new aspect of EMPA, an SGLT2 inhibitor, by assessing its effects on arrhythmias. It is believed that this study will also provide information on the underlying mechanism of SGLT2 inhibitors in general and lead to novel medical treatment for patients with cardiovascular disease and an ICD and T2DM. 


\section{Limitations}

There are several limitations to this study. First, small sample sizes and short study periods may result in inaccurate evaluation of the primary endpoint. However, as mentioned previously, this research remains important as further investigation of the mechanism of action of SGLT2 inhibitors is necessary, in particular in terms of medical treatment for patients with cardiovascular disease and an ICD and T2DM.

Second, the distribution of underlying heart disease among ICD patients in Japan is different from that in Western countries [50]. Coronary artery disease is a major cause of ICD therapy in Western countries, whereas non-ischemic heart diseases, including myocardial disease and idiopathic VF, are the major causes for ICD therapy in Japan. Differences in ethnicity and underlying heart disease may make a difference in the effect of EMPA on sympathetic nervous activity; therefore, a study in an Asian (Japanese) population remains worth conducting.

Third, it is difficult to completely balance underlying heart disease and ICD indications (primary or secondary prevention). These are related to the frequency of recurrence of arrhythmias. However, randomization with LVEF as a factor will help to maintain the balance between groups. Any significant differences in baseline patient characteristics will be included as independent variables in the adjustment of the Poisson regression analysis by the generalized linear model. In addition, the self-control study design may help to minimize the effects of confounding factors often associated with the patient's characteristics. The recurrence of arrythmias should remain stable in each patient unless the patient undergoes therapeutic intervention or progression of their disease. Consequently, patients with the following events that may alter or interfere with the probability of arrhythmia recurrence will be excluded: alteration of antiarrhythmic drug; catheter ablation for ventricular arrhythmia; coronary revascularization; open-heart surgery; development of coronary artery disease; stroke or transient ischemic stroke; seizure; infection requiring hospitalization; or cardiac failure requiring hospitalization.

Finally, tight glycemic control has the potential to lead to increased arrhythmias. Therefore, patients with a history of hypoglycemic episodes within the previous 6 months will not be included in the study.

\section{ACKNOWLEDGEMENTS}

The members of the EMPA-ICD investigators are Site 1: Tachikawa Medical Center Tachikawa General Hospital. Investigators: Masaaki Okabe, Minoru Takahashi, Hitoshi Kitazawa, Yoshio Ikeda, Satoru Fujita, Koichi Fuse, Akimitsu Nasuno, Akinori Sato, Sho Yuasa, Yusuke Ota, Sonoka Goto, Ryota Ando. Site 2: Kitasato University Hospital. Investigators: Shinichi Niwano, Hidehira Fukaya, Jun Kishihara, Jun Oikawa, Naruya Ishizue, Yuki Shirakawa, Shunsuke Ishii, Takeru Nabeta. Site 3: Kumamoto University Hospital. Investigators: Kenichi Tsujita, Hiroaki Kawano, Koichi Kaikita, Hirofumi Soejima, Eiichiro Yamamoto, Satoru Suzuki, Kenji Sakamoto, Taishi Nakamura, Hiroki Usuku, Satoshi Araki, Yuichiro Arima, Seiji Takashio, Hisanori Kanazawa, Daisuke Sueta, Tadashi Hoshiyama, Koichiro Fujisue, Miwa Ito, Fumi Oike, Ryota Sato, Masafumi Takae, Masato Nishi, Kenshi Yamanaga, Takashi Komorita, Yusei Kawahara, Mami Morioka, Yuji Kubota. Site 4: Gunma Prefectural Cardiovascular Center. Investigators: Shigeto Naito, Kohki Nakamura, Takehito Sasaki. Site 5: Kokura Memorial Hospital. Investigators: Kenji Ando, Kenichi Hiroshima, Michio Nagashima, Masato Fukunaga, Jun Hirokami. Site 6: National Cerebral and Cardiovascular Center. Investigators: Takashi Noda, Yuko Inoue, Kouhei Ishibashi, Mitsuru Wada, Tsukasa Kamakura, Kenichiro Yamagata, Koji Miyamoto, Kenzaburo Nakajima, Nobuhiko Ueda, Kiminori Hosoda, Hisashi Makino, Yoko Ohata, Tamiko Tamanaha, Ryo Koezuka, Masaki Matsubara, Mariko Harada Shiba, Cheol Son, Tsutomu Tomita, Michio 
Noguchi. Site 7: Saitama Medical University International Medical Center. Investigators: Ritsushi Kato, Yoshie Nakajima, Yoshifumi Ikeda, Kenta Tsutsui. Site 8: Sakakibara Heart Institute. Investigators: Junichi Nitta, Jun Umemura, Kanki Inoue. Site 9: Sapporo Medical University School of Medicine. Investigators: Tetsuji Miura, Daigo Nagahara. Site 10: Jichi Medical University Saitama Medical Center. Investigators: Tomio Umemoto, Yusuke Ugata, Shingo Yamamoto, Naoyuki Akashi, Takeshi Mitsuhashi. Site 11: Jichi Medical University School of Medicine. Investigators: Takahiro Komori, Tomoyuki Kabutoya, Kazuomi Kario. Site 12: Department of Cardiovascular Medicine, Chiba University Graduate School of Medicine. Investigators: Yusuke Kondo, Yoshio Kobayashi. Site 13: University of Tsukuba. Investigators: Masaki Ieda, Miyako Igarashi, Hiro Yamasaki, Yuki Komatsu, Akira Kimata. Site 14: Tokyo Women's Medical University. Investigators: Nobuhisa Hagiwara, Morio Shoda, Atsushi Suzuki. Site 15: Nagoya University Graduate School of Medicine. Investigators: Toyoaki Murohara, Yasuya Inden.. Site 16: Niigata City General Hospital. Investigators: Kazuyoshi Takahashi, Yukio Hosaka. Site 17: Niigata University Medical \& Dental Hospital. Investigators: Shinya Fujiki, Kenichi Iijima, Ippei Shimizu, Tohru Minamino. Site 18: Hirosaki University Hospital. Investigators: Hirofumi Tomita, Shingo Sasaki, Daisuke Horiuchi, Yuji Ishida, Takahiko Kinjo, Kimitaka Nishizaki. Site 19: Fukushima Medical University Hospital. Investigators: Yasuchika Takeishi, Takashi Kaneshiro, Shinya Yamada, Naoko Hijioka. Site 20: Hokkaido University Graduate School of Medicine. Investigators: Toshihisa Anzai, Masaya Watanabe, Taro Koya, Motoki Nakao .Site 21: Nippon Medical School. Investigators: Wataru Shimizu, Yu-ki Iwasaki, Kenji Yodogawa, Hiroshi Hayashi, Yoshiaki Kubota, Oka Eiichiro. Site 22: Kanazawa University Hospital. Investigators: Takeshi Kato, Masayuki Takamura, Hiroshi Furusho, Kenshi Hayashi, Toyonobu Tsuda, Satoru Niwa, Keisuke Usuda. Site 23: Shiga University of Medical Science. Investigators: Yoshihisa Nakagawa, Tomoya Ozawa, Yusuke Okuyama. Site 24: Department of Cardiovascular Medicine, Nagasaki University
Graduate School of Biomedical Sciences. Investigators: Koji MAEMURA, Satoki FUKAE, Yoshiyuki DOI, Shuji ARAKAWA. Site 25: University of Fukui Hospital. Investigators: Hiroshi Tada, Kanae Hasegawa, Moe Mukai. Site 26: Yamagata University Hospital. Investigators: Masafumi Watanabe, Takanori Arimoto, Daisuke Kutsuzawa. Site 27: Yamaguchi University Graduate School of Medicine. Investigators: Masakazu Fukuda, Yasuhiro Yoshiga, Takayoshi Kato, Masafumi Yano. Site 28: Iwate Medical University Hospital. Investigators: Shingen Owada, Reisuke Yoshizawa, Takashi Komatsu, Yohei Sawa. Site 29: Kyoto University Hospital. Investigators: Takeshi Kimura, Yugo Yamashita. Site 30: Niigata Prefectural Central Hospital. Investigators: Takashi Saikawa, Tohru Watanabe, Hiromi Kayamori, Hiroshi Onoda, Takayuki Kumaki, Sho Hirayama. Site 31: Juntendo University Hospital. Investigators: Hidemori Hayashi, Haruna Tabuchi, Tohru Minamino. Site 32: Juntendo University Urayasu Hospital. Investigators: Takashi Tokano, Fuminori Odagiri.

Funding. This study and the journal's Rapid Service Fee were funded by Nippon Boehringer Ingelheim and Eli Lilly and Company. These companies did not have a role in the study design, data collection and analysis, preparation of the manuscript or the decision to publish the findings of the study.

Authorship. All named authors meet the International Committee of Medical Journal Editors (ICMJE) criteria for authorship for this article, take responsibility for the integrity of the work as a whole, and have given their approval for this version to be published.

Authorship Contributions. All authors were involved in the study planning and operation. SF participated in the study design and drafted the manuscript. KI contributed to the conception and design of the study. TT and NK performed sample size calculations. $\mathrm{HY}$ and $\mathrm{KO}$ participated in data collection and allocation. T Minamino designed and supervised the study, and wrote the manuscript. All authors read and approved the final manuscript for publication. 
Disclosures. Shinya Fujiki, Kenichi Iijima, Masaaki Okabe, Shinichi Niwano, Kenichi Tsujita, Shigeto Naito, Kenji Ando, Ritsushi Kato, Junichi Nitta, Takeshi Mitsuhashi, Kazuomi Kario, Masaki Ieda, Kazuyoshi Takahashi, Hirofumi Tada, Yoshihisa Nakagawa, Takeshi Kimura, Hisako Yoshida, Keiko Ota, Takahiro Tanaka, Daisuke Izumi and Ippei Shimizu have nothing to disclose. Kengo Kusano has a family member that serves on the board of directors of Boehringer Ingelheim. Tetsuji Miura has received honorariums from Boehringer Ingelheim. Yusuke Kondo has received honorariums from Boehringer Ingelheim. Nobuhisa Hagiwara has received contributions from Boehringer Ingelheim. Toyoaki Murohara has received contributions from Boehringer Ingelheim, and honorariums from Boehringer Ingelheim and Eli Lilly and Company. Hirofumi Tomita has received contributions and honorariums from Boehringer Ingelheim. Yasuchika Takahashi has received honorariums from Boehringer Ingelheim. Toshihisa Anzai has received contributions from Boehringer Ingelheim. Wataru Shimizy has received honorariums from Boehringer Ingelheim. Masafumi Watanabe has received honorariums from Boehringer Ingelheim. Takeshi Kato has received honorariums from Boehringer Ingelheim. Masafumi Yano has received honorariums from Boehringer Ingelheim, and Eli Lilly and Company. Koji Maemura has received contributions and honorariums from Boehringer Ingelheim. Koichi Node received honorariums from Boehringer Ingelheim, and Eli Lilly and Company. Yoshifusa Aizawa received honorariums from Boehringer Ingelheim. Tohru Minamino discloses research funds and remuneration for lectures from Boehringer Ingelheim and Eli Lilly and Company.

Compliance with Ethics Guidelines. The study protocol was reviewed and approved by the Certified Review Board of the Niigata University Graduate School of Medicine (certification no. CRB3180025) and registered by the jRCT in February 2019 (ID jRCTs031180120). This study is being conducted in accordance with the guidelines of the Declaration of Helsinki and in compliance with the Clinical Trial
Act, a Japanese law designed to ensure that researchers monitor, adhere to the study criteria, and assess and manage any conflicts of interest. After the initial screening, each patient was given a thorough explanation of the trial plan, and before inclusion in the study, each patient provided signed informed consent forms.

Data Availability. The datasets generated during and/or analyzed during the current study are available from the corresponding author on reasonable request.

Open Access. This article is licensed under a Creative Commons Attribution-NonCommercial 4.0 International License, which permits any non-commercial use, sharing, adaptation, distribution and reproduction in any medium or format, as long as you give appropriate credit to the original author(s) and the source, provide a link to the Creative Commons licence, and indicate if changes were made. The images or other third party material in this article are included in the article's Creative Commons licence, unless indicated otherwise in a credit line to the material. If material is not included in the article's Creative Commons licence and your intended use is not permitted by statutory regulation or exceeds the permitted use, you will need to obtain permission directly from the copyright holder. To view a copy of this licence, visit http://creativecommons.org/licenses/bync/4.0/.

\section{REFERENCES}

1. Moss AJ, Hall WJ, Cannom DS, et al. Improved survival with an implanted defibrillator in patients with coronary disease at high risk for ventricular arrhythmia. Multicenter Automatic Defibrillator Implantation Trial Investigators. $\mathrm{N}$ Engl J Med. 1996;335:1933-40.

2. Moss AJ, Zareba W, Hall WJ, et al. Prophylactic implantation of a defibrillator in patients with myocardial infarction and reduced ejection fraction. N Engl J Med. 2002;346:877-83.

3. Goldenberg I, Gillespie J, Moss AJ, et al. Long-term benefit of primary prevention with an 
implantable cardioverter-defibrillator: an extended 8-year follow-up study of the Multicenter Automatic Defibrillator Implantation Trial II. Circulation. 2010;122:1265-71.

4. Bardy GH, Lee KL, Mark DB, et al. Amiodarone or an implantable cardioverter-defibrillator for congestive heart failure. N Engl J Med. 2005;352:225-37.

5. Desai AS, Fang JC, Maisel WH, Baughman KL. Implantable defibrillators for the prevention of mortality in patients with nonischemic cardiomyopathy: a meta-analysis of randomized controlled trials. JAMA. 2004;292:2874-9.

6. Golwala H, Bajaj NS, Arora G, Arora P. Implantable cardioverter-defibrillator for nonischemic cardiomyopathy: an updated meta-analysis. Circulation. 2017;135:201-3.

7. Barakat AF, Saad M, Elgendy AY, et al. Primary prevention implantable cardioverter defibrillator in patients with non-ischaemic cardiomyopathy: a meta-analysis of randomised controlled trials. BMJ Open. 2017;7:e016352.

8. Antiarrhythmics versus Implantable Defibrillators (AVID) Investigators. A comparison of antiarrhythmic-drug therapy with implantable defibrillators in patients resuscitated from near-fatal ventricular arrhythmias. N Engl J Med. 1997;337:1576-1583.

9. Kuck KH, Cappato R, Siebels J, Ruppel R. Randomized comparison of antiarrhythmic drug therapy with implantable defibrillators in patients resuscitated from cardiac arrest : the Cardiac Arrest Study Hamburg (CASH). Circulation. 2000;102:748-54.

10. Connolly SJ, Gent M, Roberts RS, et al. Canadian implantable defibrillator study (CIDS): a randomized trial of the implantable cardioverter defibrillator against amiodarone. Circulation. 2000;101: 1297-302.

11. Noda T, Kurita T, Nitta T, et al. Significant impact of electrical storm on mortality in patients with structural heart disease and an implantable cardiac defibrillator. Int J Cardiol. 2018;255:85-91.

12. Al-Khatib SM, Stevenson WG, Ackerman MJ, et al. 2017 AHA/ACC/HRS guideline for management of patients with ventricular arrhythmias and the prevention of sudden cardiac death. Circulation. 2018;138:e272-e391.

13. Priori SG, Blomstrom-Lundqvist C, Mazzanti A, et al. ESC Guidelines for the management of patients with ventricular arrhythmias and the prevention of sudden cardiac death: The Task Force for the Management of Patients with Ventricular Arrhythmias and the Prevention of Sudden Cardiac Death of the European Society of Cardiology (ESC).
Endorsed by: Association for European Paediatric and Congenital Cardiology (AEPC). Eur Heart J. 2015;36:2793-867.

14. Poole JE, Johnson GW, Hellkamp AS, et al. Prognostic importance of defibrillator shocks in patients with heart failure. $\mathrm{N}$ Engl J Med. 2008;359: 1009-177.

15. Daubert JP, Zareba W, Cannom DS, et al. Inappropriate implantable cardioverter-defibrillator shocks in MADIT II: frequency, mechanisms, predictors, and survival impact. J Am Coll Cardiol. 2008;51: 1357-65.

16. Seidl K, Hauer B, Schwick NG, Zahn R, Senges J. Comparison of metoprolol and sotalol in preventing ventricular tachyarrhythmias after the implantation of a cardioverter/defibrillator. Am J Cardiol. 1998;82:744-8.

17. American Diabetes Association. Standards of medical care in diabetes-2019 abridged for primary care providers. Clin Diabetes. 2019;37:11-34.

18. Davies MJ, D'Alessio DA, Fradkin J, et al. Management of hyperglycaemia in type 2 diabetes, 2018. A consensus report by the American Diabetes Association (ADA) and the European Association for the Study of Diabetes (EASD). Diabetologia. 2018;2018(61):2461-98.

19. Ruwald $\mathrm{MH}$, Zareba W, Jons C, et al. Influence of diabetes mellitus on inappropriate and appropriate implantable cardioverter-defibrillator therapy and mortality in the Multicenter Automatic Defibrillator Implantation Trial-Reduce Inappropriate Therapy (MADIT-RIT) Trial. Circulation. 2013;128: 694-701.

20. Zinman B, Wanner C, Lachin JM, et al. Empagliflozin, cardiovascular outcomes, and mortality in type 2 diabetes. N Engl J Med. 2015;373:2117-288.

21. Ferrannini E, Baldi S, Frascerra S, et al. Shift to fatty substrate utilization in response to sodium-glucose cotransporter 2 inhibition in subjects without diabetes and patients with type 2 diabetes. Diabetes. 2016;65:1190-5.

22. Newman JC, Verdin E. Ketone bodies as signaling metabolites. Trends Endocrinol Metab. 2014;25: 42-52.

23. Won YJ, Lu VB, Puhl HL 3rd, Ikeda SR. beta-Hydroxybutyrate modulates N-type calcium channels in rat sympathetic neurons by acting as an agonist for the G-protein-coupled receptor FFA3. J Neurosci. 2013;33:19314-25.

24. Harris PA, Taylor R, Minor BL, et al. The REDCap consortium: building an international community 
of software platform partners. J Biomed Inform. 2019;95:103208.

25. Harris PA, Taylor R, Thielke R, et al. Research electronic data capture (REDCap) - a metadata-driven methodology and workflow process for providing translational research informatics support. J Biomed Inform. 2009;42:377-81.

26. Haneda M, Noda $M$, Origasa $H$, et al. Japanese clinical practice guideline for diabetes 2016. Diabetol Int. 2018;9:1-45.

27. Chow E, Bernjak A, Williams S, et al. Risk of cardiac arrhythmias during hypoglycemia in patients with type 2 diabetes and cardiovascular risk. Diabetes. 2014;63:1738-47.

28. Cryer PE. Glucose counterregulation in man. Diabetes. 1981;30:261-4.

29. Paramore DS, Fanelli CG, Shah SD, Cryer PE. Hypoglycemia per se stimulates sympathetic neural as well as adrenomedullary activity, but, unlike the adrenomedullary response, the forearm sympathetic neural response is not reduced after recent hypoglycemia. Diabetes. 1999;48:1429-36.

30. Ogurtsova K, da Rocha Fernandes JD, Huang Y, et al. IDF Diabetes Atlas: global estimates for the prevalence of diabetes for 2015 and 2040. Diabetes Res Clin Pract. 2017;128:40-50.

31. Seshasai SRK, Kaptoge S, Thompson A, et al. Diabetes mellitus, fasting glucose, and risk of causespecific death. N Engl J Med. 2011;364:829-41.

32. Tancredi M, Rosengren A, Svensson AM, et al. Excess mortality among persons with type 2 diabetes. N Engl J Med. 2015;373:1720-32.

33. Fox CS, Coady S, Sorlie PD, et al. Trends in cardiovascular complications of diabetes. JAMA. 2004;292:2495-9.

34. Lee CD, Folsom AR, Pankow JS, Brancati FL. Cardiovascular events in diabetic and nondiabetic adults with or without history of myocardial infarction. Circulation. 2004;109:855-60.

35. Cho E, Rimm EB, Stampfer MJ, Willett WC, Hu FB. The impact of diabetes mellitus and prior myocardial infarction on mortality from all causes and from coronary heart disease in men. J Am Coll Cardiol. 2002;40:954-60.
36. Albert CM, Chae CU, Grodstein F, et al. Prospective study of sudden cardiac death among women in the United States. Circulation. 2003;107:2096-101.

37. Jouven X, Lemaitre RN, Rea TD, et al. Diabetes, glucose level, and risk of sudden cardiac death. Eur Heart J. 2005;26:2142-7.

38. Targher G, Bertolini L, Zenari L, et al. Diabetic retinopathy is associated with an increased incidence of cardiovascular events in type 2 diabetic patients. Diabet Med. 2008;25:45-50.

39. Siscovick DS, Sotoodehnia N, Rea TD, et al. Type 2 diabetes mellitus and the risk of sudden cardiac arrest in the community. Rev Endocr Metab Disord. 2010;11:53-9.

40. Neal B, Perkovic V, Mahaffey KW, et al. Canagliflozin and cardiovascular and renal events in type 2 diabetes. N Engl J Med. 2017;377:644-57.

41. Wiviott SD, Raz I, Bonaca MP, et al. Dapagliflozin and cardiovascular outcomes in type 2 diabetes. N Engl J Med. 2019;380:347-57.

42. Butler J, Hamo CE, Filippatos G, et al. The potential role and rationale for treatment of heart failure with sodium-glucose co-transporter 2 inhibitors. Eur J Heart Fail. 2017;19:1390-400.

43. Scheen AJ. Reappraisal of the diuretic effect of empagliflozin in the EMPA-REG OUTCOME trial: comparison with classic diuretics. Diabetes Metab. 2016;42:224-33.

44. Davidenko JM, Cohen L, Goodrow R, Antzelevitch C. Quinidine-induced action potential prolongation, early afterdepolarizations, and triggered activity in canine Purkinje fibers. Effects of stimulation rate, potassium, and magnesium. Circulation. 1989;79:674-86.

45. Ring A, Brand T, Macha S, et al. The sodium glucose cotransporter 2 inhibitor empagliflozin does not prolong QT interval in a thorough QT (TQT) study. Cardiovasc Diabetol. 2013;12:70.

46. Buxton AE, Lee KL, DiCarlo L, et al. Electrophysiologic testing to identify patients with coronary artery disease who are at risk for sudden death. Multicenter Unsustained Tachycardia Trial Investigators. N Engl J Med. 2000;342:1937-45.

47. Ferrannini E, Mark M, Mayoux E. CV protection in the EMPA-REG OUTCOME Trial: a "thrifty substrate" hypothesis. Diabetes Care. 2016;39:1108-14. 
48. Aubert G, Martin OJ, Horton JL, et al. The failing heart relies on ketone bodies as a fuel. Circulation. 2016;133:698-705.

49. Bedi KC Jr, Snyder NW, Brandimarto J, et al. Evidence for intramyocardial disruption of lipid metabolism and increased myocardial ketone utilization in advanced human heart failure. Circulation. 2016;133:706-16.

50. JCS Joint Working Group. Guidelines for nonpharmacotherapy of cardiac arrhythmias (JCS 2011). Circ J. 2013;77:249-274. 